\title{
Outcomes from COVID-19 across the range of frailty: excess mortality in fitter older people
}

\author{
Amy Miles ${ }^{1}$ D $\cdot$ Thomas E. Webb $^{1}$ D $\cdot$ Benjamin C. Mcloughlin ${ }^{1}$ D $\cdot$ Imran Mannan $^{1}$ (D) Arshad Rather ${ }^{1} \cdot$ Paul Knopp $^{1} \cdot$ \\ Daniel Davis ${ }^{1,2} \mathbb{D}$
}

Received: 22 May 2020 / Accepted: 2 July 2020 / Published online: 18 July 2020

(c) The Author(s) 2020

\section{Key summary points}

Aim To describe associations between frailty, ethnicity, socioeconomic position and mortality in a cohort of older patients presenting to hospital with COVID-19.

Findings Frailty did not appear to be associated with mortality rates after COVID-19, though an interaction was evident indicating much larger excess mortality in fitter, compared with frailer patients.

Message Frailty may not be a good measure of prognosis in COVID-19 and different mechanisms may underlie pathways to death depending on pre-morbid frailty.

\begin{abstract}
Purpose Our aim was to quantify the mortality from COVID-19 and identify any interactions with frailty and other demographic factors.

Methods Hospitalised patients aged $\geq 70$ were included, comparing COVID-19 cases with non-COVID-19 controls admitted over the same period. Frailty was prospectively measured and mortality ascertained through linkage with national and local statutory reports.

Results In 217 COVID-19 cases and 160 controls, older age and South Asian ethnicity, though not socioeconomic position, were associated with higher mortality. For frailty, differences in effect size were evident between cases (HR 1.02, 95\% CI 0.93-1.12) and controls (HR 1.99, 95\% CI 1.46-2.72), with an interaction term (HR 0.51, 95\% CI 0.37-0.71) in multivariable models.

Conclusions Our findings suggest that (1) frailty is not a good discriminator of prognosis in COVID-19 and (2) pathways to mortality may differ in fitter compared with frailer older patients.
\end{abstract}

Keywords COVID-19 · Frailty $\cdot$ Mortality $\cdot$ Epidemiology

\section{Introduction}

While it is clear that mortality from SARS-CoV-2 infection (COVID-19) increases with age [1-3], the association between frailty and mortality is not well understood $[4,5]$.

Amy Miles and Thomas E. Webb contributed equally.

Daniel Davis

daniel.davis@ucl.ac.uk

1 Department of Medicine for the Elderly, University College London Hospitals NHS Foundation Trust, London WC1E 7HB, UK

2 Department of Population Science and Experimental Medicine, MRC Unit for Lifelong Health and Ageing at UCL, 1-19 Torrington Place, London, UK This relationship has clinical implications as the National Institute for Health and Care Excellence (NICE) guidelines in England and Wales recommend the integration of a frailty assessment into algorithms used to guide decisions including admission to critical care [6]. Furthermore, other demographic factors relevant to mortality such as ethnicity or socioeconomic position have yet to be comprehensively described in relation to COVID-19 [7, 8]. 
Our aim was to investigate the relationship between frailty, ethnicity, socioeconomic position and mortality in a cohort of older patients presenting to hospital with COVID19, in order to: (1) quantify the mortality from COVID-19; (2) identify any interactions with frailty and other demographic factors in this population.

\section{Methods}

\section{Participants}

Patients admitted to an urban teaching hospital aged $\geq 70$ were included if they tested positive for SARS-CoV-2 by combined throat and high-nasal swab on reverse-transcriptase polymerase chain reaction or if there was high clinical suspicion (on the basis of clinical, imaging and laboratory results, as determined by specialist infectious diseases physicians) for COVID-19 up until 23rd April 2020. During the pandemic, the index of suspicion for SARS-CoV-2 infection was very high, so each older person needing hospitalisation was systematically assessed for COVID-19. Therefore, the control group comprised patients aged $\geq 70$ who had been admitted within the same time period who reliably did not have COVID-19.

\section{Outcome}

Our primary outcome was all-cause mortality determined up until 13th May 2020. Deaths occurring outside of hospital were captured through daily updates on the NHS Spine, a collection of local and national databases and systems containing demographic information.

\section{Exposures}

Frailty was quantified by the Clinical Frailty Scale (CFS) score [9] capturing patients' clinical state 2 weeks prior to admission. This was assessed prospectively by the admitting clinical team, though all scores were reviewed by specialist geriatricians. Socioeconomic position was estimated through the Index of Multiple Deprivation (IMD) (along with Health, Income, Education sub-indices) which is an ecological measure determined by home postcodes [10]. Ethnicity was self-reported in hospital administrative data.

\section{Ethics approvals}

These analyses were conducted as part of a service evaluation project and individual consent was not necessary as determined by the NHS Health Research Authority (HRA), the regulatory body for medical research for England, UK. The HRA has the Research Ethics Service as one of its core functions and they determined the project was exempt from the need to obtain approval from an NHS Research Ethics Committee [11].

\section{Statistical analysis}

Differences in continuous or categorical variables were assessed using $t$ tests and $\chi^{2}$ tests, respectively. Cox proportional hazards models estimated differences in survival between COVID-19 cases and non-COVID-19 controls. Frailty was considered a continuous variable, ethnicity was classified into South Asian, Black, White, Mixed, Other, and deciles of IMD $(1=$ most advantaged; $10=$ most disadvantaged) and its sub-indices were used in univariable and multivariable models. Interactions between Clinical Frailty Score and COVID-19 status were assessed. Statistical significance was determined at $p<0.05$. Post-estimation procedures included Schoenfeld residuals to test heteroskedasticity. Stata 14.1 (StataCorp, Texas, USA) was used for all analyses.

\section{Results}

A total of 217 COVID-19 cases and 160 non-COVID-19 controls were identified. In COVID-19 cases, the mean age was 80.0 (SD 6.8) (range 70-99) years (Table 1). The majority of cases were men $(n=134,62 \%)$ or of white ethnicity $(n=138,63 \%)$. There was a normal distribution of clinical frailty scores and the median Index of Multiple Deprivation decile was 4 (IQR 3, 6). There were no significant differences in age, ethnicity, Index of Multiple Deprivation decile, or Clinical Frailty Scale score between cases and controls (Table 1). Median length of stay was 9 (IQR 4,7) and 4 (IQR $2,8)$ in COVID-19 and controls, respectively $(p<0.01)$.

In univariable models, COVID-19, older age and South Asian ethnicity were associated with higher mortality, though no measure of socioeconomic position demonstrated any association (Table 2). For frailty, differences in effect size were evident between cases (HR 1.02, 95\% CI $0.93-1.12, p=0.71)$ and controls (HR $1.99,95 \%$ CI $1.46-2.72, p<0.01)$. In the multivariable model, these relationships remained consistent: age (HR 1.04, 95\% CI $1.01-1.07, p<0.01$ ), South Asian ethnicity (HR 1.13, 95\% CI 1.13-3.51, $p=0.02$ ) (Table 2).

The different associations with frailty according to COVID-19 status was confirmed by demonstrating an interaction term (HR 0.51, 95\% CI 0.37-0.71, $p<0.01$ ). The coefficient direction suggests that mortality is proportionally higher in fitter patients. Estimated in this way, the overall mortality attributable to COVID-19 was extremely high in this population (HR 213, 95\% CI 24.6-1841, $p<0.01$ ). When plotting mutually adjusted survival curves, tertiles of 
Table 1 Patient characteristics of study participants by COVID-19 status

\begin{tabular}{|c|c|c|c|}
\hline & $\begin{array}{l}\text { COVID- } \\
19 \text { cases } \\
(n=217)\end{array}$ & Controls $(n=160)$ & $p$ \\
\hline Age (SD) & $80.0(7.6)$ & $81.4(6.8)$ & 0.06 \\
\hline $\operatorname{Sex}(\%) \mathrm{M}$ & $134(61.8)$ & $78(48.8)$ & 0.01 \\
\hline Ethnicity (\%) & & & 0.92 \\
\hline South Asian & $16(7.4)$ & $9(5.6)$ & \\
\hline Black & $16(7.4)$ & $15(9.4)$ & \\
\hline White & $138(63.6)$ & $103(64.4)$ & \\
\hline Mixed & $19(8.8)$ & $13(8.1)$ & \\
\hline Unknown & $28(12.9)$ & $20(12.5)$ & \\
\hline $\begin{array}{l}\text { Median IMD Decile } \\
\text { (IQR) }\end{array}$ & $4(3,6)$ & $4(2,6)$ & 0.09 \\
\hline $\begin{array}{l}\text { Median Health and Dis- } \\
\text { ability Decile (IQR) }\end{array}$ & $6(4,8)$ & $6(4,8)$ & 0.14 \\
\hline CFS $(\%)$ & & & 0.37 \\
\hline 1 & $4(1.9)$ & $2(1.3)$ & \\
\hline 2 & $32(14.9)$ & $14(8.8)$ & \\
\hline 3 & $32(14.9)$ & $24(15.0)$ & \\
\hline 4 & $32(14.9)$ & $36(22.5)$ & \\
\hline 5 & $25(11.6)$ & $25(15.6)$ & \\
\hline 6 & $41(19.1)$ & $26(16.3)$ & \\
\hline 7 & $32(14.9)$ & $24(15.0)$ & \\
\hline 8 & $16(7.4)$ & $9(5.6)$ & \\
\hline 9 & $1(0.5)$ & $0(0.0)$ & \\
\hline Death & $111(51.2)$ & $22(13.8)$ & $<0.01$ \\
\hline
\end{tabular}

$S D$ standard deviation, IQR interquartile range, CFS Clinical Frailty Scale
CFS showed distinct trajectories in non-COVID-19 controls, not at all apparent in COVID-19 cases (Fig. 1). In keeping with the interaction parameter, differences were most stark in fitter patients (CFS 1-3) but less so in frailer ones (CFS 7-9).

\section{Discussion}

In this population of older admissions to a central London hospital, frailty did not appear to be associated with mortality rates after COVID-19. In addition, ecological measures of socioeconomic position were not associated with death, though there was some evidence of geater risk in South Asian compared with White populations. Associations with mortality in those with and without COVID-19 demonstrated much larger excess mortality in fitter, compared with frailer patients. Taken together, our findings suggest that (1) frailty is not a good discriminator of prognosis in COVID-19 and (2) pathways to mortality may differ in fitter compared with frailer older patients.

Our results should be treated with caution. Data were collected from a single site, albeit a large teaching hospital at the first peak of the COVID-19 pandemic. For a proportion (21\%), ethnicity was either mixed or undetermined, perhaps reflecting a casemix specific to London. Furthermore, ecological measures of socioeconomic position will be less reliable compared with individual factors, and the index of multiple deprivation may not relate to health outcomes as well in London residents [12], or influence mortality once admitted to secondary care. Our results are only applicable
Table 2 Univariable and multivariable analysis of the effect of cohort characteristics on mortality in COVID-19

\begin{tabular}{|c|c|c|c|c|c|c|c|c|}
\hline \multirow[b]{3}{*}{ Age } & \multicolumn{4}{|c|}{ Univariable models } & \multicolumn{4}{|c|}{ Multivariable model } \\
\hline & \multirow{2}{*}{$\begin{array}{l}\mathrm{HR} \\
1.03\end{array}$} & \multicolumn{2}{|c|}{$95 \% \mathrm{CI}$} & \multirow{2}{*}{$\frac{p}{0.01}$} & \multirow{2}{*}{$\begin{array}{l}\mathrm{HR} \\
1.04\end{array}$} & \multicolumn{2}{|c|}{$95 \% \mathrm{CI}$} & \multirow{2}{*}{$\frac{p}{<0.01}$} \\
\hline & & 1.01 & 1.06 & & & 1.01 & 1.07 & \\
\hline Sex & 1.41 & 0.99 & 2.01 & 0.06 & 1.39 & 0.96 & 2.01 & 0.08 \\
\hline \multicolumn{9}{|l|}{ Ethnicity } \\
\hline South Asian & 2.08 & 1.20 & 3.6 & 0.01 & 2.00 & 1.13 & 3.51 & 0.02 \\
\hline Black & 1.12 & 0.60 & 2.1 & 0.72 & 1.30 & 0.70 & 2.51 & 0.38 \\
\hline White & [Ref] & & & & [Ref] & & & \\
\hline Mixed & 1.00 & 0.53 & 1.89 & 0.99 & 0.93 & 0.49 & 1.77 & 0.83 \\
\hline Unknown & 0.85 & 0.48 & 1.49 & 0.57 & 0.96 & 0.54 & 1.70 & 0.88 \\
\hline IMD & 1.02 & 0.95 & 1.11 & 0.54 & 1.00 & 0.93 & 1.08 & 0.98 \\
\hline Income & 1.04 & 0.97 & 1.11 & 0.27 & & & & \\
\hline Education & 1.01 & 0.94 & 1.09 & 0.76 & & & & \\
\hline Health & 1.02 & 0.95 & 1.09 & 0.56 & & & & \\
\hline CFS & 1.12 & 1.02 & 1.23 & 0.02 & 1.88 & 1.37 & 2.59 & $<0.01$ \\
\hline COVID-19 & 4.40 & 2.78 & 6.97 & $<0.01$ & 213 & 24.63 & 1842 & $<0.01$ \\
\hline $\begin{array}{l}\text { COVID-19 } \times \text { CFS } \\
\text { interaction }\end{array}$ & & & & & 0.51 & 0.37 & 0.71 & $<0.01$ \\
\hline
\end{tabular}

$H R$ hazard ratio, $C I$ confidence interval, IMD Index of Multiple Deprivation, CFS Clinical Frailty Scale 
Fig. 1 Kaplan-Meier curves showing 60 days survival by tertiles of Clinical Frailty Scale (CFS) and COVID-19 status

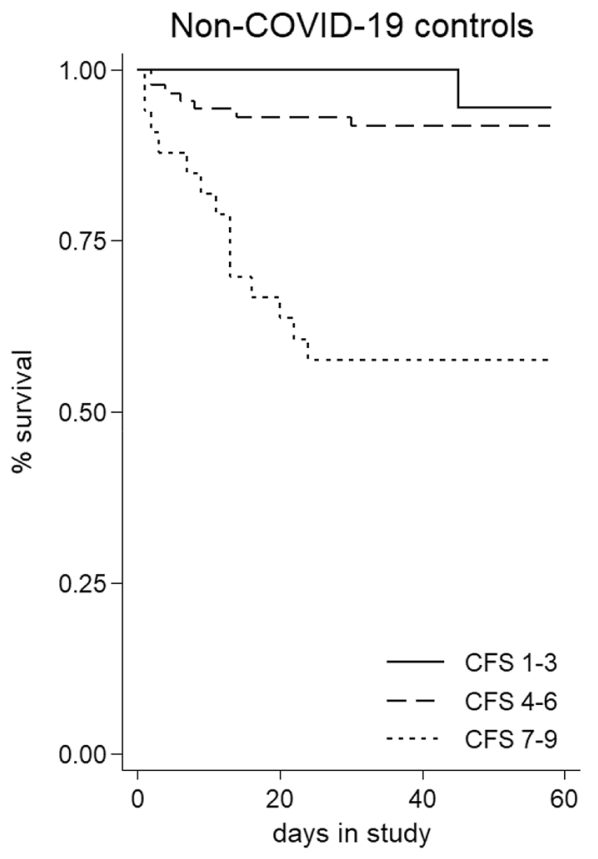

to hospitalised patients, and some selection bias might arise from different indications for presenting to secondary care in COVID-19 patients versus those without respiratory symptoms (our controls). We used the Clinical Frailty Scale as the instrument recommended by NICE, but other frailty measures may have had different associations with mortality in the context of COVID-19. Nonetheless, our data have the advantage of specialist assessments of COVID-19 status and frailty, as well as accurate statutory reporting of dates of death.

These findings add to emerging reports quantifying the relationship between frailty and mortality in COVID-19. In another London hospitalised cohort, crude deaths in COVID-19 were higher in patients who were frailer (median Clinical Frailty Scale score of 5 versus 4, $p=0.01$ ) [13]. Other UK case series have shown that patients who died without ventilatory support had a median Clinical Frailty Scale score of 7 [14]. To date, most studies are describing mortality without reference to a contemporaneous nonCOVID-19 population, which would obscure the interaction apparent in our data. In this respect, our findings are most consistent with comparable data from Leicester which also show no association between frailty and mortality in COVID-19 [15].

Our findings have two major implications. First, if frailty states in COVID-19 are not associated with mortality, then this has only limited value as a consideration in older people who may require ventilatory support. This is in contrast to the central NICE guidance that recommends a frailty assessment as the first step in the assessment for critical care. Second, an interaction between COVID-19 and frailty implies that different pathways to death could be at play. In general, the pathophysiology described in COVID-19 patients in critical care indicates substantial immune hyperactivation [16]. However, given survival in the CFS range 7-9 was similar in cases and controls, this may reflect death from COVID-19 is occurring in the same way as for other common illness and immune hyperactivation is unlikely to be a significant feature in this group. One might speculate that older people with frailty have pre-existing immunesenescence such that they are unable to mount excess immune responses and may be otherwise by dying from the direct effects of viral infection.

While COVID-19 clearly confers substantial mortality in older people, we show that this risk may arise for different reasons depending on pre-morbid frailty. Further work should consider other outcomes after COVID-19, particularly cognitive and physical function. If baseline frailty and associated immunesenescence influences the subsequent inflammatory response, this hints that different therapeutic strategies might be needed across the spectrum of frailty.

Author contributions AM, TW, BM, PK: collected the primary data; DD: undertook the statistical analyses and had oversight of the project; AM and TW: drafted the first version of the manuscript. All authors contributed to revision and intellectual content of the final submission

Funding Daniel Davis is funded through a Wellcome Intermediate Clinical Fellowship (WT107467). 


\section{Compliance with ethical standards}

Conflict of interest The authors declare that they have no conflict of interest.

Ethics approval These analyses were conducted as part of a service evaluation project and individual consent was not necessary as determined by the NHS Health Research Authority.

Open Access This article is licensed under a Creative Commons Attribution 4.0 International License, which permits use, sharing, adaptation, distribution and reproduction in any medium or format, as long as you give appropriate credit to the original author(s) and the source, provide a link to the Creative Commons licence, and indicate if changes were made. The images or other third party material in this article are included in the article's Creative Commons licence, unless indicated otherwise in a credit line to the material. If material is not included in the article's Creative Commons licence and your intended use is not permitted by statutory regulation or exceeds the permitted use, you will need to obtain permission directly from the copyright holder. To view a copy of this licence, visit http://creativecommons.org/licenses/by/4.0/.

\section{References}

1. CDC Covid-Response Team (2020) Severe outcomes among patients with coronavirus disease 2019 (COVID-19)-United States, February 12-March 16, 2020. MMWR Morb Mortal Wkly Rep 69(12):343-346. https://doi.org/10.15585/mmwr.mm6912e2

2. Wu Z, McGoogan JM (2020) Characteristics of and important lessons from the coronavirus disease 2019 (COVID-19) outbreak in China: summary of a report of 72314 cases from the Chinese Center for Disease Control and Prevention. JAMA J Am Med Assoc. https://doi.org/10.1001/jama.2020.2648

3. Banerjee A, Pasea L, Harris S, Gonzalez-Izquierdo A, Torralbo A, Shallcross L et al (2020) Estimating excess 1-year mortality associated with the COVID-19 pandemic according to underlying conditions and age: a population-based cohort study. Lancet. https ://doi.org/10.1016/S0140-6736(20)30854-0

4. Lian J, Jin X, Hao S, Cai H, Zhang S, Zheng L et al (2019) Analysis of epidemiological and clinical features in older patients with Corona Virus Disease 2019 (COVID-19) out of Wuhan. Clin Infect Dis. https://doi.org/10.1093/cid/ciaa242

5. Zhou F, Yu T, Du R, Fan G, Liu Y, Liu Z et al (2020) Clinical course and risk factors for mortality of adult inpatients with COVID-19 in Wuhan, China: a retrospective cohort study. Lancet 395(10229):1054-1062. https://doi.org/10.1016/S0140 $-6736(20) 30566-3$
6. National Institute of Health and Care Excellence (2020) COVID19 rapid guideline: critical care in adults. NICE, London

7. Williamson E, Walker AJ, Bhaskaran KJ, Bacon S, Bates C, Morton CE et al (2020) OpenSAFELY: factors associated with COVID-19-related hospital death in the linked electronic health records of 17 million adult NHS patients. medRxiv. https://doi. org/10.1101/2020.05.06.20092999

8. Patel AP, Paranjpe MD, Kathiresan NP, Rivas MA, Khera AV (2020) Race, socioeconomic deprivation, and hospitalization for COVID-19 in English participants of a National Biobank. medRxiv. https://doi.org/10.1101/2020.04.27.20082107

9. Rockwood K, Song X, MacKnight C, Bergman H, Hogan DB, McDowell I et al (2005) A global clinical measure of fitness and frailty in elderly people. Can Med Assoc J 173(5):489-495

10. McLennan D, Noble S, Noble M, Plunkett E, Wright G, Gutacker N (2019) The English indices of deprivation 2019: technical report. 2019

11. https://www.hra.nhs.uk/about-us/committees-and-services/resand-recs. Accessed 16 July 2020

12. Steel N, Ford JA, Newton JN, Davis ACJ, Vos T, Naghavi M et al (2018) Changes in health in the countries of the UK and 150 English Local Authority areas 1990-2016: a systematic analysis for the Global Burden of Disease Study 2016. Lancet 392(10158):1647-1661. https://doi.org/10.1016/S0140 $-6736(18) 32207-4$

13. Brill SE, Jarvis H, Ozcan E, Burns T, Warraich R, Amani L et al (2020) COVID-19: a retrospective cohort study with focus on the over-80s and hospital-onset disease. medRxiv. https://doi. org/10.1101/2020.05.11.20097790

14. Baker KF, Hanrath AT, Schim van der Loeff I, Tee SA, Capstick R, Marchitelli G et al (2020) COVID-19 management in a UK NHS Foundation Trust with a High Consequence Infectious Diseases centre: a detailed descriptive analysis. medRxiv. https://doi. org/10.1101/2020.05.14.20100834

15. Owen RK, Conroy SP, Taub N, Jones W, Bryden D, Pareek M, Faull C, Abrams K, Davis D, Baneree J (2020) Comparing associations between frailty and mortality in hospitalised older adults with or without COVID-19 infection: a retrospective observational study using electronic health records. Age Ageing. https://doi. org/10.1093/ageing/afaa167

16. Tay MZ, Poh CM, Renia L, MacAry PA, Ng LFP (2020) The trinity of COVID-19: immunity, inflammation and intervention. Nat Rev Immunol. https://doi.org/10.1038/s41577-020-0311-8

Publisher's Note Springer Nature remains neutral with regard to jurisdictional claims in published maps and institutional affiliations. 